\title{
13.1
}

\section{Прозрачное радиоэкранирующее покрытие, полученное при помощи самоорганизованного шаблона}

\author{
(C) А.С. Воронин ${ }^{1,2}$, Ю.В. Фадеев ${ }^{1}$, И.В. Говорун ${ }^{3,4}$, А.С. Волошин ${ }^{2-4}$, И.А. Тамбасов ${ }^{3}$, М.М. Симунин ${ }^{1,2,4}$, \\ C.B. Хартов ${ }^{1}$ \\ ${ }^{1}$ ФИЦ „Красноярский научный центр СО РАН““, Красноярск, Россия \\ ${ }^{2}$ Сибирский федеральный университет, Красноярск, Россия \\ ${ }^{3}$ Институт физики им. Л.В. Киренского СО РАН, Красноярск, Россия \\ ${ }^{4}$ Сибирский университет науки и технологий им. акад. М.Ф. Решетнёва, Красноярск, Россия \\ E-mail: a.voronin1988@mail.ru
}

Поступило в Редакцию 3 августа 2020 г.

В окончательной редакции 24 ноября 2020г.

Принято к публикации 24 ноября 2020 г.

\begin{abstract}
Представлена простая и доступная технология получения тонкопленочного прозрачного радиоэкранирующего материала. Материал представляет собой серебряное микросетчатое покрытие, полученное при помощи самоорганизованного шаблона. Представлены результаты исследования радиоэкранирующих свойств данных покрытий в $X$ - и $K$-диапазонах. Показано, что микросетчатое покрытие с поверхностным сопротивлением $6.8 \Omega / \mathrm{sq}$ и интегральным оптическим пропусканием $83.6 \%$ характеризуется эффективностью экранирования $28.4 \mathrm{~dB}$ на частоте $8 \mathrm{GHz}$, что соответствует экранированию $99.85 \%$ излучения. Основным механизмом экранирования радиоизлучения микросетчатыми покрытиями является отражение.
\end{abstract}

Ключевые слова: самоорганизованный шаблон, микросетчатое покрытие, экранирование электромагнитного излучения.

DOI: 10.21883/PJTF.2021.05.50674.18496

В 80-х годах XX века была показана принципиальная возможность перехвата побочного радиоизлучения от монитора персонального компьютера (ПЭМИН-утечки) с его последующей декодировкой. В литературе методика получила название „перехват ван Эйка“ [1]. Для борьбы с паразитным радиоизлучением монитора и других прозрачных объектов используется экранирование. Для электромагнитного экранирования подобного рода объектов необходимо использовать такие материалы, которые сочетают оптическую прозрачность более $80 \%$ с поверхностным сопротивлением $R_{s}$ менее $10 \Omega / \mathrm{sq}$. Низкое значение поверхностного сопротивления позволяет материалу эффективно экранировать электромагнитное излучение в радиодиапазоне не менее чем на 20-30 dB или экранировать 99-99.9\% мощности падающего излучения. Основными материалами, применяемыми для радиоэкранирования прозрачных объектов, являются прозрачные проводящие оксиды, такие как ITO, AZO и т.д. [2]; тонкие пленки одностенных углеродных нанотрубок $[3,4]$; композитные пленки на основе нанотрубок и проводящих полимеров [5]; пленки металлических нанопроволок [6,7]; литографические сетчатые микро- и наноструктуры [8]. В настоящей работе в качестве прозрачного радиоэкранирующего материала предлагается использовать серебряное микросетчатое (МКС) покрытие, полученное при помощи самоорганизованного шаблона.

Процесс формирования самоорганизованного шаблона подробно описан в [9]. В настоящей работе в качестве материала для формирования самоорганизо- ванного шаблона был выбран яичный белок. Раствор яичного белка с концентрацией желтка $3 \mathrm{ml} / 1$ наносился методом Meyer rod на РET-подложки толщиной $50 \mu \mathrm{m}$. Толщина слоя яичного белка $t_{w}$ составляла $35.56 \mu \mathrm{m}$ (Meyer rod \#14) — образец Ag МКС № 1 - и $71.1 \mu \mathrm{m}$ (Meyer rod \#28) - образец Ag МКС № 2. После нанесения жидкая пленка высушивалась при комнатной температуре $21^{\circ} \mathrm{C}$ и влажности $\sim 50 \%$. В процессе сушки происходит растрескивание пленки яичного белка, что и является конечным этапом формирования самоорганизованного шаблона [9].

Напыление серебра на самоорганизованные шаблоны производилось магнетронным методом на установке Emitech K575XD (Quorum Technologies Ltd, UK). Толщина пленки серебра на пластине-спутнике составляла $\sim 200 \mathrm{~nm}$. После напыления серебра шаблон отмывался в дистиллированной воде [9]. Морфология микросетчатых покрытий была исследована методом сканирующей электронной микроскопии (СЭМ) при помощи микроскопа Hitachi TM3000 (Japan), ускоряющее напряжение $15 \mathrm{kV}$.

Спектральная зависимость оптического пропускания серебряных микросетчатых покрытий измерялась в диапазоне 400-700 nm, прибор Shimadzu UV-3600 (Japan). Вольт-амперные характеристики покрытий были измерены с помощью прибора IPPP-1 (Belarus). Удельное поверхностное сопротивление покрытий вычислялось из тангенса угла наклона вольт-амперных характеристик.

Эффективность экранирования электромагнитного излучения $(S E)$ полученных образцов определялась вол- 
Таблица 1. Значение толщины слоя яичного белка $t_{w}$, коэффициента заполнения $F F$, оптической прозрачности $T_{o p t}$ и поверхностного сопротивления $R_{s}$ для образцов $\mathrm{Ag}$ МКС № 1 и $\mathrm{Ag} \mathrm{MKC} \mathrm{№} 2$

\begin{tabular}{c|c|c|c|c}
\hline Тип покрытия & $t_{w}, \mu \mathrm{m}$ & $F F, \%$ & $T_{o p t}(550 \mathrm{~nm}), \%$ & $R_{s}, \Omega / \mathrm{sq}$ \\
\hline Ag МКС № 1 & 35.56 & 10.2 & 90.2 & 11.2 \\
Ag МКС № 2 & 71.1 & 15.5 & 83.6 & 6.8
\end{tabular}

новодным методом в $X$-диапазоне $(8-12 \mathrm{GHz})$ и $K$-диапазоне $(18-26 \mathrm{GHz})$. Для этого при помощи векторного анализатора цепей (VNA) Rohde\&Schwarz ZVA 50 (Germany) проводилось измерение параметров отраженной и прошедшей электромагнитной волны $\left(S_{11}, S_{21}\right)$. Исследуемые образцы серебряных микросетчатых покрытий имели прямоугольную форму (размер $3 \times 4 \mathrm{~cm}$ ). Образцы полностью перекрывали волноводное окно размером $23 \times 10 \mathrm{~mm}$ для $X$-диапазона и $11 \times 5.5 \mathrm{~mm}$ для $K$-диапазона.

СЭМ-изображения покрытий $\mathrm{Ag}$ МКС № 1 и $\mathrm{Ag}$ МКС № 2 представлены на рис. 1, $a$ и $b$. Согласно статистической обработке СЭМ-изображений, средний размер ячейки составляет $63 \pm 22 \mu \mathrm{m}$ для $\mathrm{Ag} \mathrm{MKC}$ № 1 и $67 \pm 25 \mu \mathrm{m}$ для $\mathrm{Ag}$ МКС № 2. Величина ширины трещины составляет $3.3 \pm 0.8 \mu \mathrm{m}$ для $\mathrm{Ag}$ МКС № 1 и $5.4 \pm 1.4 \mu \mathrm{m}$ для $\mathrm{Ag}$ МКС № 2 . Коэффициент заполнения поверхности металлом (fill factor, $F F)$ может быть вычислен согласно выражению $F F=\left(1-(p-w)^{2} / p^{2}\right) \cdot 100 \%$, где $p-$ средний размер ячейки шаблона, а $w-$ средняя ширина трещины. На рис. 1, с приведены спектральные зависимости оптического пропускания покрытий $\mathrm{Ag}$ МКС № 1 и Ag МКС № 2 в диапазоне 400-700 nm.

В табл. 1 представлены основные данные о геометрических и оптоэлектрических характеристиках полученных микросетчатых покрытий. Как видно из таблицы, увеличение толщины слоя яичного белка образца приводит к увеличению коэффициента заполнения и, как следствие, к уменьшению поверхностного сопротивления и оптической прозрачности.

Экранирование электромагнитного излучения происходит за счет поглощения и отражения. Следует отметить, что в случае толстых пленочных структур также необходимо учитывать множественные отражения [10]. Коэффициент отражения $(R)$, передачи $\left(T_{\mathrm{SHF}}\right)$ и поглощения $(A)$ мощности можно выразить как

$$
\begin{gathered}
R=\frac{P_{r}}{P_{i}}=\left(10^{\left(0.1 S_{11}\right)}\right) \cdot 100 \%, \\
T_{\mathrm{SHF}}=\frac{P_{t}}{P_{i}}=\left(10^{\left(0.1 S_{21}\right)}\right) \cdot 100 \%, \\
A=100 \%-T_{\mathrm{SHF}}-R,
\end{gathered}
$$

где $P_{i}, P_{r}, P_{t}-$ мощности падающей, отраженной и прошедшей волны, параметры матрицы рассеяния $S_{11}$ и $S_{21}$ следует брать в dB. Полную эффективность экранирования можно характеризовать исходя из величины

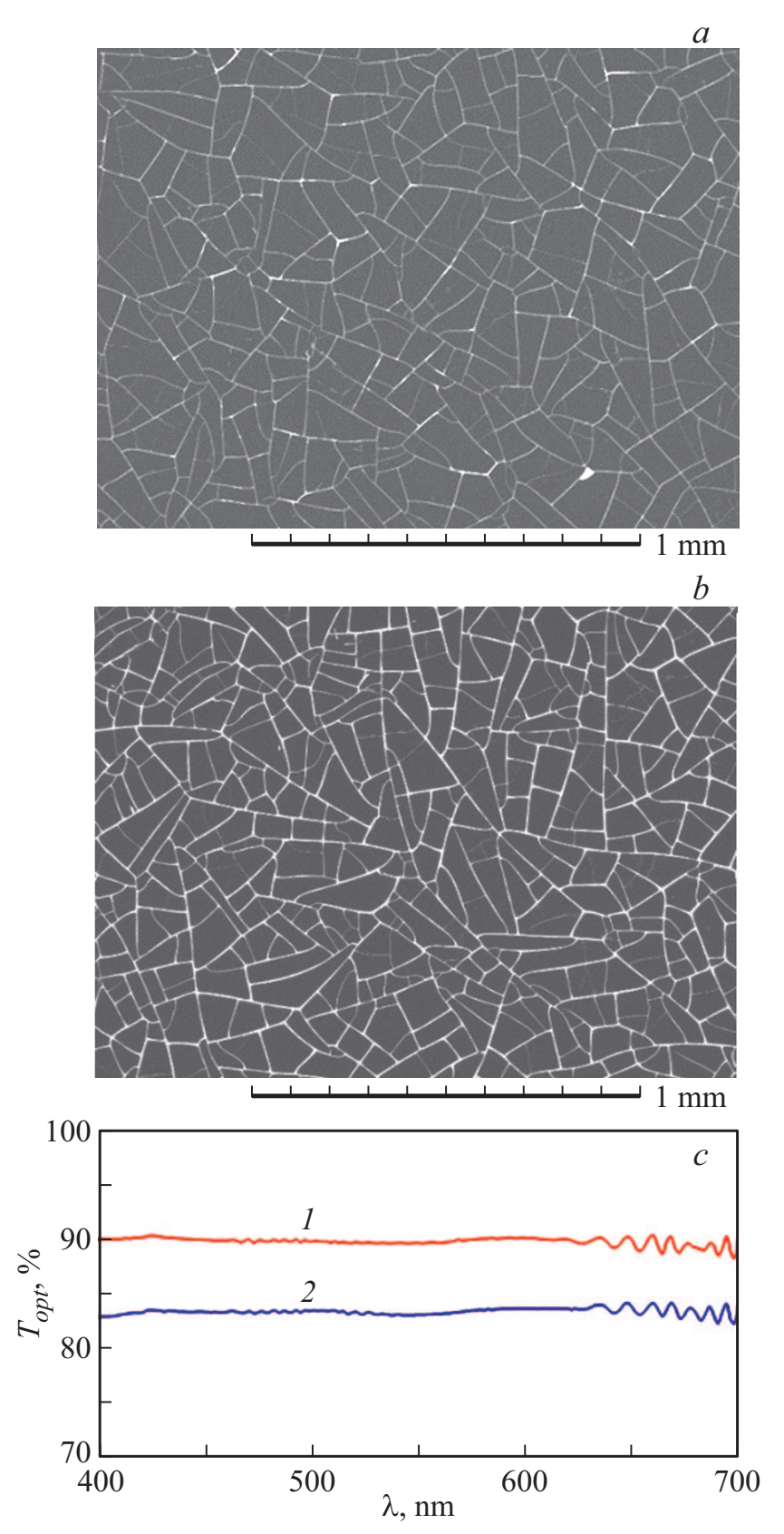

Рис. 1. СЭМ-изображения $\mathrm{Ag}$ МКС № $1(a)$ и $\mathrm{Ag}$ МКС № 2 $(b) ; c$ - оптическое пропускание образцов $\mathrm{Ag}$ МКС № 1 (1) и $\mathrm{Ag}$ МКС № 2 (2).

коэффициента прохождения согласно выражению

$$
S E=-10 \lg T_{\mathrm{SHF}} .
$$


Таблица 2. Значения параметра $S E$, коэффициента передачи $T_{\mathrm{SHF}}$ и коэффициента отражения $R$ для покрытий Аg MКС № 1 и Ag МКС № 2 на граничных частотах

\begin{tabular}{c|c|c|c|c|c|c}
\hline $\begin{array}{c}\text { Тип } \\
\text { покрытия }\end{array}$ & $\begin{array}{c}S E(8 \mathrm{GHz}), \\
\mathrm{dB}\end{array}$ & $\begin{array}{c}T_{\mathrm{SHF}} \\
(8 \mathrm{GHz}), \%\end{array}$ & $\begin{array}{c}R \\
(8 \mathrm{GHz}), \%\end{array}$ & $\begin{array}{c}S E \\
(26 \mathrm{GHz}), \mathrm{dB}\end{array}$ & $\begin{array}{c}T_{\mathrm{SHF}} \\
(26 \mathrm{GHz}), \%\end{array}$ & $\begin{array}{c}R \\
(26 \mathrm{GHz}), \%\end{array}$ \\
\hline Ag МКС № 1 & 23.1 & 0.49 & 89.3 & 15.5 & 2.82 & 80.5 \\
Ag МКС № 2 & 28.4 & 0.15 & 93.1 & 18.5 & 1.4 & 86.1
\end{tabular}
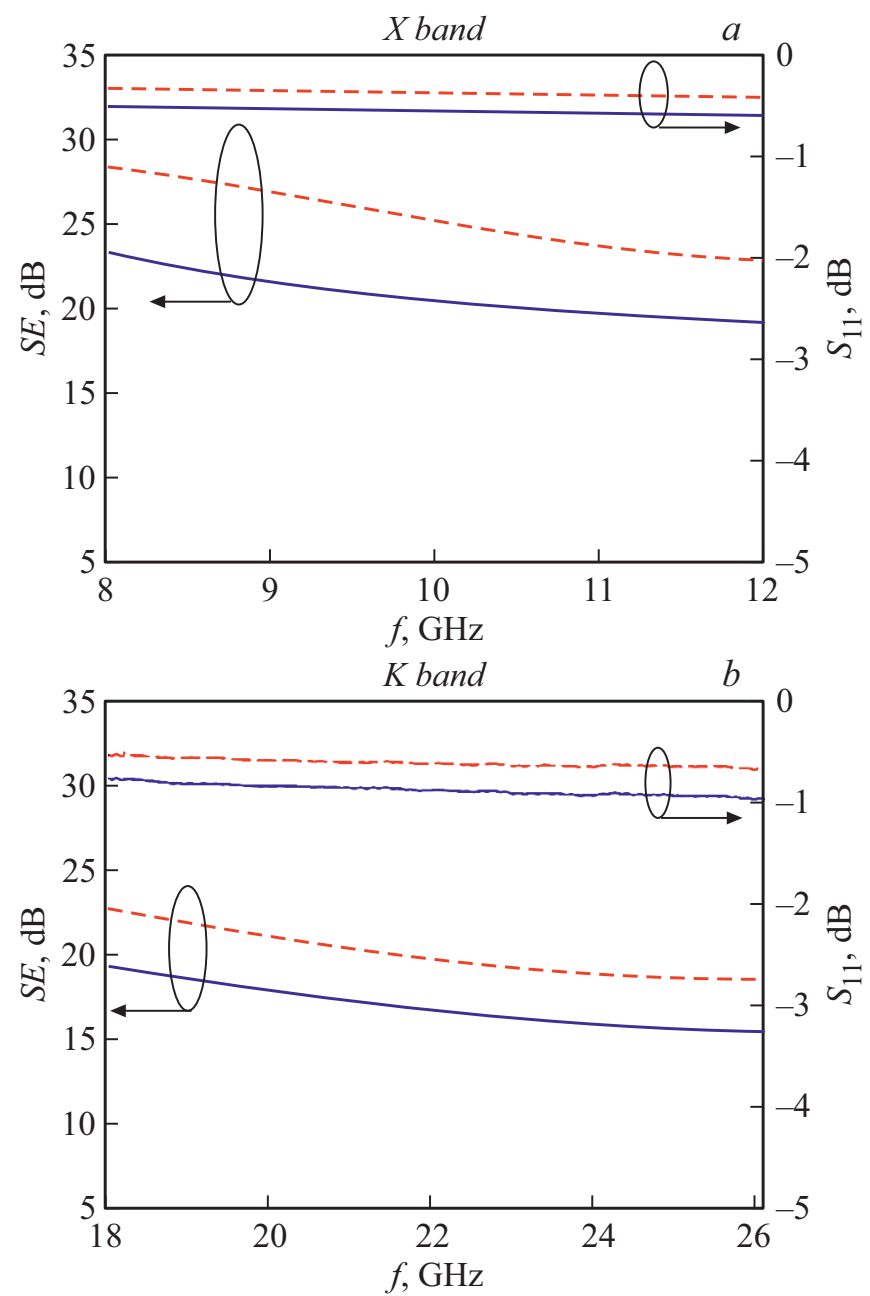

Рис. 2. Частотные зависимости эффективности экранирования $S E$ и коэффициента отражения $S_{11}$ в $X$ - $(a)$ и $K$-диапазоне $(b)$. Сплошные линии - $\mathrm{Ag}$ МКС № 1 , штриховые - $\mathrm{Ag}$ МКС № 2.

Частотные зависимости эффективности экранирования $S E$ и коэффициента отражения $S_{11}$ для образцов $\mathrm{Ag}$ МКС № 1 и Ag МКС № 2 в $X$ - и $K$-диапазонах приведены на рис. 2. Как видно, для исследуемых образцов с ростом частоты величина $S E$ незначительно уменьшается, а значит, эффективность экранирования электромагнитного излучения падает. При этом уменьшение $S E$ с ростом частоты сопровождается уменьшением $S_{11}$. Это проявляется в том, что уменьшается доля падающей мощности, которая отражается от образцов. Так, для $\mathrm{Ag}$ МКС № 1 в $X$-диапазоне величина $S E$ плавно уменьшается от $23.4 \mathrm{~dB}$ на частоте $8 \mathrm{GHz}$ до $19.2 \mathrm{~dB}$ на частоте $12 \mathrm{GHz}$, а в $K$-диапазоне $S E$ принимает значения 19.2 и $15.4 \mathrm{~dB}$ на частотах 18 и $26 \mathrm{GHz}$ соответственно. Уменьшение $S E$ и $S_{11}$ с ростом частоты связано с частотной зависимостью поверхностного сопротивления металла, на основе которого изготовлен образец. Как известно, для обычных проводников с металлическим типом проводимости активная часть их поверхностного сопротивления с увеличением частоты растет, причем пропорционально корню из частоты [10].

Во всех рассматриваемых частотных диапазонах образец Ag MКС № 2 эффективнее экранирует электромагнитное излучение, чем образец $\mathrm{Ag}$ МКС № 1. Так, на частоте $10 \mathrm{GHz} S E$ составляет 20 и $25 \mathrm{~dB}$ для $\mathrm{Ag}$ МКС № 1 и $\mathrm{Ag}$ МКС № 2 соответственно. Это объясняется тем, что поверхностное сопротивление $\left(R_{s}\right)$ для образца Ag МКС № 2 меньше, чем для Ag МКС № 1 (табл. 1).

Величины $S E$, а также коэффициентов передачи $T_{\mathrm{SHF}}$ и отражения $R$ для покрытий $\mathrm{Ag}$ МКС № 1 и $\mathrm{Ag} \mathrm{MKC}$ № 2 на границах исследуемых диапазонов приведены в табл. 2.

Используя формулы (1)-(3), можно оценить часть падающей мощности, которая поглощается в образце. Например, на частоте $8 \mathrm{GHz}$ для $\mathrm{Ag}$ МКС № 2 $S E=28.4 \mathrm{~dB}$, а $S_{11}=-0.31 \mathrm{~dB}$. В данном случае через исследуемый образец проходит всего $0.15 \%$ падающей мощности. Следует заметить, что такое значительное ослабление обусловлено тем, что подавляющая часть $(93.1 \%)$ падающей мощности отражается от образца.

Таким образом, в работе представлены результаты исследования радиоэкранирующих свойств серебряных микросетчатых покрытий в $X$ - и $K$-диапазонах. Микросетчатое покрытие характеризуется величиной $S E$, равной $28.4 \mathrm{~dB}$ на частоте $8 \mathrm{GHz}$, что соответствует экранированию 99.85\% излучения. Предлагаемые покрытия могут быть использованы для экранирования прозрачных объектов, таких как дисплеи компьютеров и смартфонов, а также при остеклении зданий в случае высоких требований к защите информации.

\section{Благодарности}

Исследования методом сканирующей электронной микроскопии и спектрофотометрии выполнены на обо- 
рудовании Красноярского регионального центра коллективного пользования ФИЦ КНЦ СО РАН.

\section{Конфликт интересов}

Авторы заявляют, что у них нет конфликта интересов.

\section{Список литературы}

[1] W. van Eck, Comput. Security, 4 (4), 269 (1985). doi.org/10.1016/0167-4048(85)90046-X

[2] Y.-J. Choi, K.-M. Kang, H.-S. Lee, H.-H. Park, Thin Solid Films, 583, 226 (2015). doi.org/10.1016/j.tsf.2015.04.001

[3] H. Xu, S.M. Anlage, L. Hu, G. Gruner, Appl. Phys. Lett., 90 (18), 3119 (2007). doi.org/10.1063/1.2734897

[4] K.F. Akhmadishina, I.I. Bobrinetskii, R.A. Ibragimov, I.A. Komarov, A.M. Malovichko, V.K. Nevolin, V.A. Petukhov, Inorgan. Mater., 50 (1), 23 (2014). DOI: $10.7868 / \mathrm{S} 0002337 \mathrm{X} 14010011$

[5] А.С. Воронин, М.М. Симунин, Ф.С. Иванченко, А.В. Шиверский, Ю.В. Фадеев, И.А. Тамбасов, И.В. Немцев, А.А. Мацынин, С.В. Хартов, Письма в ЖТФ, 43 (17), 12 (2017). DOI: 10.21883/PJTF.2017.17.44941.16702

[6] I. Anoshkin, I. Nefedova, I.S. Nefedov, D. Lyubchenko, A. Nasibulin, A. Raisanen, Micro \& Nano Lett., 11 (7), 343 (2016). DOI: 10.1049/mnl.2015.0582

[7] M. Hu, J. Gao, Y. Dong, K. Li, G. Shan, S. Yang, R.K.-Y. Li, Langmuir, 28 (18), 7101 (2012). doi.org/10.1021/la300720y

[8] Y. Han, J. Lin, Y. Liu, H. Fu, Y. Ma, P. Jin, J. Tan, Sci. Rep., 6, 25601 (2016). doi.org/10.1038/srep25601

[9] А.С. Воронин, М.М. Симунин, Ю.В. Фадеев, Ф.С. Иванченко, Д.В. Карпова, И.А. Тамбасов, С.В. Хартов, Письма в ЖТФ, 45 (7), 59 (2019).

DOI: 10.21883/PJTF.2019.07.47542.17626

[10] Y. Liu, J. Tan, Prog. Electromagn. Res., 140, 353 (2013).

DOI: 10.2528/PIER13050312 